\title{
Laserterapia de baixa potência aplicada em lesões labiais de paciente em condição
}

\section{grave: relato de caso}

\author{
Low-power laser therapy applied to lip lesions in a critically ill patient: case report \\ Terapia con láser de baja potencia aplicada a las lesiones labiales en un paciente crítico: reporte de
}

caso

Recebido: 14/01/2022 | Revisado: 18/01/2022 | Aceito: 20/01/2022 | Publicado: 22/01/2022

Luiza Elias Ota

ORCID: https://orcid.org/0000-0003-4561-5423

Universidade Federal de Mato Grosso do Sul, Brasil

E-mail: eliasota.luiza@gmail.com

Gabriel Umbelino Santana

ORCID: https://orcid.org/0000-0003-4651-5491

Hospital Universitário Maria Aparecida Pedrossian, Brasil

E-mail:gaielsantanna@gmail.com

Yuri Nejaim

ORCID: https://orcid.org/0000-0002-0442-2601

Universidade Federal de Mato Grosso do Sul, Brasil

E-mail: ynejaim@ @otmail.com

Ellen Cristina Gaetti-Jardim

ORCID: https://orcid.org/0000-0003-2471-465X Hospital Universitário Maria Aparecida Pedrossian, Brasi

Universidade Federal de Mato Grosso do Sul, Brasil

E-mail:ellen.jardim@ufms.br

\begin{abstract}
Resumo
A associação entre a Síndrome da Imunodeficiência Adquirida e as manifestações bucais, embora já bastante conhecida, ainda apresenta situações desafiadoras. Seja ocasionada ou potencializada pela doença, as alterações bucais são uma realidade e para tanto é objetivo deste trabalho relatar e discutir o caso de um paciente de 45 anos, sexo masculino, internado no Hospital Universitário Maria Aparecida Pedrossian com diagnóstico de doença pelo vírus da imunodeficiência humana (HIV), epilepsia e tetraplegia, de evolução grave. Fazendo uso de ventilação mecânica e demais cuidados paliativos, o mesmo passou a apresentar úlceras e lesões sangrantes em mucosa labial, de modo que a equipe médica solicitou o parecer da odontologia hospitalar. Para o manejo dessas feridas foi estabelecido, então, o protocolo de laserterapia de baixa frequência, dividido em 05 aplicações, para propiciar uma melhor biomodulação e regeneração do tecido afetado, diminuindo, assim, a morbidade do paciente. Ao final da última sessão, os resultados foram satisfatórios, evidenciando a eficácia do tratamento coadjuvante com laser de baixa frequência em lesões orais. Mesmo com as dificuldades inerentes ao tratamento e diagnóstico do HIV e suas manifestações, diminuir a sintomatologia do paciente é também um dever da Odontologia, assim, os resultados alcançados com a terapêutica empregada foram satisfatórios e devolveram alguma qualidade de vida ao paciente neste momento tão desafiador.
\end{abstract}

Palavras-chave: HIV; Terapia com Luz de Baixa Intensidade; Manifestações Bucais.

\begin{abstract}
The association between Acquired Immunodeficiency Syndrome and oral manifestations, although already well known, still presents challenging situations. Whether caused or enhanced by the disease, oral changes are a reality and, therefore, the objective of this paper is to report and discuss the case of a 45-year-old male patient, admitted to the University Hospital Maria Aparecida Pedrossian with a diagnosis of disease caused by the immunodeficiency virus human (HIV), epilepsy and quadriplegia, with severe evolution. Making use of mechanical ventilation and other palliative care, he started to present ulcers and bleeding lesions in the labial mucosa, so the medical team requested the opinion of the hospital dentistry. For the management of these wounds, a low-frequency laser therapy protocol was then established, divided into 5 applications, to provide better biomodulation and regeneration of the affected tissue, thus reducing patient morbidity. At the end of the last session, the results were satisfactory, showing the effectiveness of the adjuvant treatment with low-frequency laser in oral lesions. Even with the difficulties inherent in the treatment and diagnosis of HIV and its manifestations, reducing the patient's symptoms is also a duty of Dentistry, thus, the results achieved with the therapy used were satisfactory and returned some quality of life to the patient at this very challenging time.
\end{abstract}

Keywords: HIV; Low-Level Light Therapy; Oral Manifestations. 


\begin{abstract}
Resumen
La asociación entre el Síndrome de Inmunodeficiencia Adquirida Y las manifestaciones orales, aunque ya es bien conocida, todavía presenta situaciones desafiantes. Ya sean provocados o potenciados por la enfermedad, los cambios bucales son una realidad Y, por ello, el objetivo de este trabajo es informar Y discutir el caso de un paciente masculino de 45 años, ingresado en el Hospital Universitario María Aparecida Pedrossian con un diagnóstico de enfermedad causada por el virus de la inmunodeficiencia humana (VIH), epilepsia Y cuadriplejía, con evolución severa. Haciendo uso de la ventilación mecánica y otros cuidados paliativos, comenzó a presentar úlceras Y lesiones hemorrágicas en la mucosa labial, por lo que el equipo médico solicitó la opinión de la odontología del hospital. Para el manejo de estas heridas se estableció entonces un protocolo de terapia con láser de baja frecuencia, dividido en 5 aplicaciones, para proporcionar una mejor biomodulación Y regeneración del tejido afectado, reduciendo así la morbilidad del paciente. Al final de la última sesión, los resultados fueron satisfactorios, mostrando la efectividad del tratamiento adyuvante con láser de baja frecuencia en las lesiones bucales. Aún con las dificultades inherentes al tratamiento Y diagnóstico del VIH y sus manifestaciones, la reducción de los síntomas del paciente también es un deber de la Odontología, por lo que los resultados obtenidos con la terapia utilizada fueron satisfactorios $\mathrm{Y}$ devolvieron algo de calidad de vida al paciente en este mismo momento tiempo desafiante.
\end{abstract}

Palabras clave: VIH; Terapia por Luz de Baja Intensidad; Manifestaciones Cutáneas.

\title{
1. Introdução
}

Com o seu advento no princípio da década de 1980, a AIDS, ou Síndrome da Imunodeficiência Adquirida, e o Vírus da Imunodeficiência Humana - HIV evidenciaram uma severa imunossupressão ao hospedeiro, apresentando inúmeras manifestações pelo corpo (Borges et al., 2013), sendo a cavidade bucal e os tecidos periorais os mais acometidos. Dentre estas, as lesões labiais, podem se apresentar na forma de eritema, fissuração e descamação do tecido, favorecendo a colonização de leveduras, sobretudo Candida albicans, e de bactérias como o Staphylococcus aureus (Neville et al., 2016; Souza et al., 2000).

Inúmeros estudos têm sido realizados abordando as manifestações orais e periorais da AIDS, uma vez que elas representam os primeiros sinais da doença (Oliveira Bezerra et al., 2021). O manejo e tratamento dessas lesões orais visam a redução da dor e o estímulo ao processo de reparo tecidual. A terapia convencional, com métodos farmacológicos, sejam eles tópicos ou sistêmicos, é uma das formas de controlar essas manifestações, sendo ainda, extremamente utilizada e difundida na literatura, por apresentar resultados satisfatórios. Entretanto, na maior parte dos casos, tal técnica não surta efeitos imediatos, promovendo redução da dor e da lesão após 3 a 5 dias de tratamento. Associado a isso, os efeitos colaterais pelo uso dessas medicações, sejam analgésicos e anti-inflamatórios, sejam antibióticos, afetam negativamente o tratamento e a adesão do paciente (Ahmed et al., 2020; Lins et al., 2010; Legouté et al., 2019; Valle et al., 2017).

Diante disso, novas maneiras de abordar e tratar essas lesões são cada vez mais pesquisadas e relatadas, com destaque, principalmente, para a terapia com laser de baixa potência ou laser terapêutico. Através de aparelhos pequenos, portáteis e com ótimo custo-benefício, feixes de luz altamente organizados, emitidos em baixas energias, promovem uma fotobiomodulação do tecido, gerando, consequentemente, uma ação analgésica, anti-inflamatória e auxiliando, ainda, o processo de reparo da ferida, atuando tanto na angiogênese quanto na proliferação celular e síntese de componentes da matriz extracelular, como as fibras colágenas (Lins et al., 2010; Leal et al., 2012).

Ainda que na literatura não exista um consenso acerca das doses e frequência das aplicações, sabe-se que os efeitos gerados, na maior parte dos casos descritos, são imediatos e visíveis após a primeira aplicação do laser, o que, somado a ausência de efeitos colaterais, tornam essa terapia uma opção extremamente viável e que deve ser levada em conta no manejo das lesões orais, principalmente em pacientes hospitalizados (Ahmed et al., 2020; Amorim dos Santos et al., 2021; Santos et al., 2018).

É válido, então, discutir o tema, abordando as mais diversas aplicações da terapia com laser na cavidade oral - em especial, os de baixa potência, assim como seus resultados, devido, principalmente ao atual cenário, em que manifestações bucais em pacientes hospitalizados tem sido cada vez mais relatadas e urge a necessidade de tratá-los de forma integrada, buscando restabelecer sua saúde o mais rápido possível.

Portanto, é objetivo relatar o caso de paciente internado no Hospital Universitário Maria Aparecida Pedrossian - 
HUMAP, em estado grave, o qual apresentou lesões em boca tratadas com laser de baixa potência como terapia coadjuvante.

\section{Metodologia e Relato de Caso}

O presente caso trata-se de um relato com fins descritivos, exploratórios e com abordagem qualitativa, a fim de mostrar sua relevância clínica e facilitar pesquisas e novos relatos com a mesma temática, sempre baseada em evidência, usando como base referencial Santana et al. (2021) e Souza et al. (2021). O trabalho recebeu anuência dos responsáveis pelo paciente após assinatura de Termo de Consentimento para participação e uso de suas imagens. Foram respeitados todos os princípios éticos na condução do tratamento do paciente condizentes com os preceitos da Declaração de Helsinque.

Assim, trata-se de paciente do sexo masculino, 45 anos, que deu entrada no Pronto Atendimento Médico do Hospital Universitário Maria Aparecida Pedrossian (HUMAP) com histórico de 6 episódios de crise convulsiva, sem uso de medicações prévias e ausência de pródromos infecciosos, evoluindo com duas paradas cardiorrespiratórias com retorno em dois ciclos de ressuscitação cardiopulmonar (RCP) e intubação orotraqueal (IOT), conforme o médico do SAMU. Ao adentrar o HUMAP, foi relatado pela acompanhante que o paciente fazia uso de Ácido Valpróico 500mg, uma vez ao dia, no período noturno, e que era HIV positivo com abandono de tratamento.

O paciente permaneceu internado em uso de Tenofovir 300mg associado à Lamivudina 300mg, Dolutegravir 50mg, Sulfametoxazol e Trimetoprima (400 + 80mg), Teicoplanina 400mg, Polimixina B 500.000UI (50mg), Amicacina 100mg/2mL e sedoanalgesia com Midazolam $50 \mathrm{mg}$, Fentanil $10 \mathrm{~mL}$, Propofol 2\% e Dexmedetomedina, sendo este em caso de grande agitação psicomotora.

Com nove dias de internação hospitalar, a IOT foi substituída pela traqueostomia. Em seguida, na avaliação da equipe de odontologia hospitalar, observou-se que o paciente apresentava lesões ulceradas em lábio inferior, de aspecto profundo e sanguinolento. Foi estabelecido, então, um protocolo de laserterapia, em 05 sessões, com intervalo de 24 horas entre elas, a fim de auxiliar o processo de cicatrização das feridas, associada a cuidados na região. No mesmo dia, foi realizada a primeira aplicação de laser e remoção da crosta sangrante, assim como higienização da região com solução de digluconato de clorexidina $0,12 \%$. A equipe de enfermagem foi orientada a respeito do cuidado na manipulação do tubo orotraqueal e sua fixação, a fim de evitar maiores danos aos tecidos peribucais.

Com 100mW de potência, utilizando 5 joules (L1D5 - Laser Duo MM Optics) de frequência, laser vermelho, a cada $1 \mathrm{~cm}^{2}$ de área do lábio foi irradiada por 50 segundos. Na segunda sessão, as melhoras já eram visíveis, as quais foram progredindo ao longo do protocolo, com evolução substancial ao final da quinta aplicação (Figura 1). 
Figura 1: Sequência das cinco sessões de laserterapia, uma aplicação ao dia.

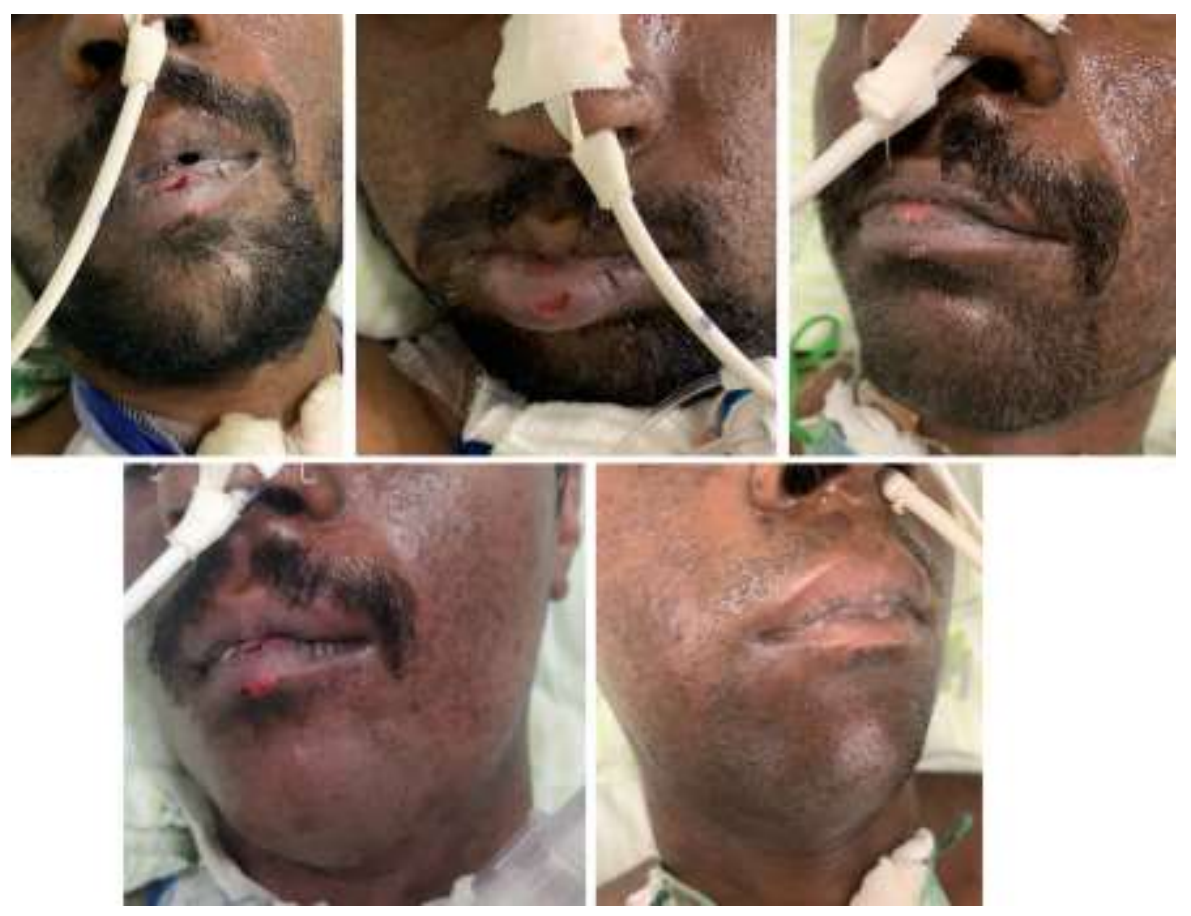

Fonte: Autores.

Ao final da última sessão do protocolo de laserterapia o paciente apresentava, clinicamente, as feridas em fase adiantada no processo de reparação tecidual, com destaque para a diminuição acentuada das crostas labiais e retorno ao aspecto normal da região. Tais resultados demonstram a eficácia do protocolo de laser de baixa frequência e a necessidade de sua difusão e utilização, a fim de auxiliar na recuperação do paciente, reduzindo sua morbidade e promovendo incremento à qualidade de vida enquanto hospitalizado.

\section{Discussão}

Muito além do tratamento de alterações teciduais, o atendimento ao paciente com HIV é de alta complexidade, tendo o HUMAP/EBSERH um centro de referência a assistência destes pacientes, provendo, por meio de uma equipe multiprofissional, o controle e tratamento de afecções desta magnitude na cavidade oral, sempre enxergando o paciente de modo integral, associando à alterações na resposta imunológica, adequação da higiene oral e nutrição, que em conjunto podem e agravam quaisquer afecções da cavidade bucal. (Walsh et al., 2006; Aas et al., 2007). Tal assertiva vai ao encontro com o caso apresentado, a melhora significativa das feridas labiais do paciente e sua completa remissão foi potencializada pelo controle sistêmico do mesmo em consonância a terapêutica local com o laser de baixa potência.

Sem aquele estigma da sentença de morte que outrora rondava o diagnóstico do HIV/AIDS, a determinação da qualidade de vida ao paciente portador do vírus é obrigação do profissional de saúde, sendo função deste a promoção de conforto ao paciente (Cecílio et al., 2020). Analisando essa sentença, então, existe uma forte relação entre bem-estar e lesões descamativas e ulceradas em áreas de grande algia e extremamente expostas, como os lábios. De tal modo, é fundamental que o profissional se renove e busque alternativas visando sempre a melhora do quadro e, consequentemente, do paciente.

Diante disso, o uso de terapias coadjuvantes, como a laserterapia de baixa frequência, tem se mostrado extremamente eficaz e vantajosa, seja por apresentar melhora nas lesões logo após a primeira aplicação do protocolo, seja por não por provocar efeitos colaterais locais e sistêmicos (Santos et al., 2018). Seu alto potencial em estimular o processo de cicatrização e reparo tecidual, além de analgesia, sem, contudo, provocar complicações clínicas, como foi exposto, ainda, por Ahmed et al. (2020) e 
Lins et al. (2010), consagra cada vez mais essa terapia na literatura, de modo que seu uso é cada vez mais difundido, seja em âmbito clínico, seja em âmbito hospitalar, sobretudo em pacientes imunocomprometidos (Rezende et al., 2020; Campos et al., 2021; Raffaele et al., 2021).

No caso relatado, optou-se pela aplicação de 5 sessões de laser de baixa potência, com frequências variadas, de modo a conseguir uma melhor biomodulação tecidual. Ao final da última aplicação, as feridas encontravam-se, cicatrizadas, tendo assim, não apenas uma melhora local, principalmente na questão dolorosa, mas, também, na parte sistêmica do paciente, por minimizar as chances de uma infecção oportunista se instalar nas feridas abertas.

O uso do laser como terapia coadjuvante mostra a sua eficácia, como também mencionado neste artigo, sendo extremamente necessária sua difusão e utilização, tanto por médicos, quanto por cirurgiões-dentistas, para que a recuperação do paciente seja cada vez mais rápida e com menos efeitos colaterais. Os resultados foram visíveis, favorecendo de forma conservadora e não invasiva a reparação tecidual e, com isso, a melhoria da sintomatologia associada (Raffaele et al., 2021).

A presença do cirurgião-dentista em ambiente hospitalar, em tese, já é algo mais que consolidado. Na prática, o atendimento ao paciente sistemicamente comprometido é uma realidade e o dentista não pode se furtar do atendimento de qualidade aos pacientes imunossuprimidos. Então, a atenção especializada, associada a uma equipe multiprofissional, sobretudo com o dentista focado na cavidade oral e tecidos faciais, buscando não somente a cura local, mas, principalmente, bons resultados sistêmicos, possibilitarão a promoção de saúde para estes pacientes. Com isso, a inspeção criteriosa por esses profissionais facilitará o diagnóstico precoce de enfermidades que se manifestam, primariamente, em boca (Borges et al., 2013).

Por fim, o uso de terapêuticas coadjuvantes, não menos importantes, como o laser de baixa potência, é muito promissor, algo também aqui evidenciado, mas, necessita de mais estudos, uma vez que a técnica ainda é recente. Assim, vem somar com os achados deste e outros relatos relevantes que em conjunto mostram uma terapêutica rápida e eficaz, com aceleração no processo de cicatrização e sem efeitos colaterais (Barros et al., 2021).

\section{Conclusão}

O caso mostrado busca ampliar os conhecimentos sobre o uso do laser de baixa potência no atendimento de pacientes imunossuprimidos e suas possíveis atuações nos tecidos peribucais. Soma-se a isso, também, a agressão local que os aparatos de ventilação mecânica podem gerar nos tecidos adjacentes em pacientes com períodos de intubação orotraqueal.

A importância da terapia coadjuvante com a laserterapia de baixa frequência no manejo dessas úlceras promove um melhor reparo tecidual, e de forma mais rápida, quando comparado as terapias convencionais. Devido as incógnitas que ainda existem, esse trabalho apresenta limitações inerentes aos relatos de caso, de modo que são necessários mais estudos e relatos acerca do tema para a consolidação estatística da melhor forma de tratamento em casos semelhantes.

\section{Agradecimentos}

O presente trabalho foi realizado com apoio da Fundação Universidade Federal de Mato Grosso do Sul -UFMS/MEC -Brasil.

\section{Referências}

Aas, J. A., Barbuto, S. M., Alpagot, T., Olsen, I., Dewhirst, F. E., \& Paster, B. J. (2007). Subgingival plaque microbiota in HIV positive patients. Journal of Clininical Periodontology. 34(3):189-195

Ahmed, M. K., Jafer, M., Nayeem, M., Moafa, I. H., Ali Q. M. F., Gopalaiah, H., et al. (2020). Low-level laser therapy and topical medications for treating aphthous ulcers: A systematic review. Journal Multidisciplinary Healthcare. 13:1595-605. https://doi.org/10.2147/JMDH.S281495.

Barros, B. F. M., Costa, C. M., \& Mattos, G. M. L. (2021). Uso do laser de baixa potência no tratamento de lesões bucais em pacientes com doenças neurológicas: Relato de caso. Research, Society and Development, v. 10, n. 6, e47110616083, 2021). 
Borges, H. C. F., Santos, K. R. R. dos, Gaetti Jardim, E. C., Cheade, M. F. M., \& Motta, E. F. da. (2013). Atenção odontológica ao paciente com hiv: relato de caso. Revista Brasileira de Ciências da Saúde. 11(35): 56-61.

Campos, L., Rezende, S. B., Simões, A., Palma, L. F., Tateno, R.Y., da Silva, R. L. \& Macedo, M. C. (2020). Photobiomodulation and photodynamic therapy for the management of oral graft-versus-host disease: A case report. Photodiagnosis and Photodynamic Therapy. 30, 101776.

Cecilio, H. P. M., Oliveira, D. S., Apostolidis, T., Dany, L., \& Oliveira, D. C. de. (2020). Time perspective, socioeconomical status and quality of life of people living with HIV. Research, Society and Development. 1. 9(8): e148985451. DOI: 10.33448/rsd-v9i8.5451.

Leal, C. T., Bezerra, A. L., \& Lemos, A. (2012). A efetividade do laser de HeNe 632,8 nm no reestabelecimento da integridade dos tecidos cutâneos em animais experimentais: revisão sistemática. Fisioterapia e Pesquisa. 19:290-6. https://doi.org/10.1590/s1809-29502012000300016.

Legouté, F., Rene-Jean, J. B., Valerie, S., Yoann, P., Delphine, C., Philippe, L., et al. (2019). Low-level laser therapy in treatment of chemoradiotherapy-induced mucositis in head and neck cancer: Results of a randomised, triple blind, multicentre phase III trial. Radiation Oncology. 14:1-11. 10.1186/s13014-019-1292-2.

Lins, R. D. A. U., Dantas, E. M., Lucena, K. C. R., Catão, M. H. C. V., Garcia, A. F. G., \& Carvalho Neto, L. G. (2010). Efeitos bioestimulantes do laser de baixa potência no processo de reparo. Anais Brasileiros de Dermatologia. 85(6):849-55.

Neville, B. W. Damm, D. D., Allen, C. M., \& Chi, A. C. (2016). Patologia oral e maxilofacial. (4a ed.), Elsevier. 912p.

Oliveira Bezerra, H. I. de, Gonzaga, A. K. G., Dias, A. C. M. da S., Oliveira, M. M. de. Silva, J. Z. e, Oliveira, P. T. de, \& Medeiros, A. M. C. de. (2021). Archives of Health Investigation. Hiperpigmentação oral em pacientes vivendo com HIV: relato de dois casos clínicos.

Raffaele, R. M., Baldo, M. E., Queiroz, P. F. de S., Tateno, R. Y., Palma, L. F., \& Campos, L. (2021). Phototherapies in the management of an ulcerative lip lesion in a patient taking multiple medications for rheumatoid arthritis. Research, Society and Development. 10(8): e50210817538. DOI: 10.33448/rsdv10i8.17538.

Raffaele, R. M., Baldo, M. E., Souza, Y. D., \& Gaetti-Jardim, E. C. Lasertherapy as an adjuvante in the repair of Chemical lesions in the mouth. Research, Society and Development. 10(15): e44101522340, 2021. DOI: 10.33448/rsd-v10i15.22340.

Rezende, S. B., Campos, L., Palma, L. F., Tateno, R. Y., Simões, A., Macedo, M. C. \& Silva, R. L. da. (2020). Photobiomodulation and antimicrobial photodynamic therapy for oral cytomegalovirus reactivation following acute graft-versus-host disease. Photodiagnosis and Photodynamic Therapy. $32,101849$.

Rezende Santana, G. U., Costa, G. P. Da., Souza, Y. D., Queiroz, P. F. De S., Raffaele, R. M., Faverani, L. P., \& Gaetti Jardim, E. C. (2021). Low-power laser in the treatment of exuberant manifestations of congenital syphilis. Research, Society and Development. 10(14): e02101421664. DOI: 10.33448/rsdv10i14.21664.

Santos, T. K. G. L., Alcoforado, L. G. C., Leitão, A. S., Vanderlei, A. C. Q. V., Carvalho, A. K. F. A., \& Vieira, A. P. S. B. (2018). Uso da laserterapia de baixa potência no tratamento de lesões orais. Revista Campo do Saber. 4:240-57.

Souza, L. B., Pereira Pinto, L., Medeiros, M. C., Araújo Jr, R. F., \& Mesquita, O. J. X. (2000). Manifestações orais em pacientes com AIDS em uma população brasileira. Pesquisa Odontológica Brasileira. 14(1): 79-85.

Souza, Y. D., Seco, A. J. L. G.,Silva, J. C. L. Da., Pereira, K. F. S., \& Jardim, E. C. G. (2021). Kaposi’s Sarcoma in an HIV and Covid-19 positive patient. Research, Society and Development. 10(11), e19101119149.

Umakanthan, S., Sahu, P., Ranade, A. V., Bukelo, M. M., Rao, J. S, Abrahao-Machado, L. F., et al. (2020). Origin, transmission, diagnosis and management of coronavirus disease 2019 (COVID-19). Postgraduate Medical Journal. 96:753-8. https://doi.org/10.1136/postgradmedj-2020-138234.

Valle, L. A., Karam, P. S. B. H., Rezende, M. L. R., Zangrando, M. S. R., \& Damante, C. A. (2017). Laser de baixa intensidade no tratamento de úlceras traumáticas e queilite angular: relatos de casos. Revista da Associação Paulista de Cirurgiões Dentistas. 71:30-4.

Walsh, M. C., Kim, N., Kadono, Y., Rho, J., Lee, S. Y., Lorenzo, J., et al. (2006). Osteoimmunology: interplay between the immune system and bone metabolism. Annual Review of Immunology. 23(1):33-63.

Wiersinga, W. J., Rhodes, A., Cheng, A. C., Peacock, S. J., \& Prescott, H. C. (2020). Pathophysiology, Transmission, Diagnosis, and Treatment of Coronavirus Disease 2019 (COVID-19): A Review. JAMA - Journal of the American Medical Association. 324:782-93. https://doi.org/10.1001/jama.2020.12839. 